\title{
Conservative Management of Chyle Leak following Thyroid Surgery
}

${ }^{1}$ Sabaretnam Mayilvaganan, ${ }^{2}$ Aromal Chekavar, ${ }^{3}$ Rakesh Kapoor, ${ }^{4}$ Amit Agarwal

\begin{abstract}
Lymphatic injury leading to chyle leak is a potential complication that may arise following thyroid surgery especially when there is a high nodal burden. Although the incidence of chyle leak post surgery is low ( 1 to $4 \%$ ), this complication can present significant challenges to both the surgeon and patient. Multiple approaches to the treatment of chyle leak have emerged, including nutrition, surgical, and pharmacological therapies. Although there are strong feelings among clinicians about the use of bowel rest, parenteral nutrition, or a low-fat enteral formula for the treatment of chyle leak, definitive evidence supporting any nonsurgical methods vs surgical approach is lacking. The lack of a clear consensus on the optimal management of chyle leaks makes this problem more challenging for the treating surgeon.
\end{abstract}

Keywords: Chyle leak, Conservative management, Thyroid surgery.

How to cite this article: Mayilvaganan S, ChekavarA, Kapoor R, Agarwal A. Conservative Management of Chyle Leak following Thyroid Surgery. World J Endoc Surg 2015;7(3):76-78.

Source of support: Nil

Conflict of interest: Sabaretnam Mayilvaganan and Aromal Chekavar have contributed equally to this manuscript.

\section{INTRODUCTION}

The lymph system includes the lymph vessels and capillaries, the thoracic duct, lymph nodes, the spleen, thymus, bone marrow and gut-associated lymphoid tissue (GALT), as well as other structures. The primary functions of the lymph system include its immunological role, the absorption of excess interstitial fluid and its return to the bloodstream, and the transport of long chain fat and fat-soluble vitamins. Chyle (from the Latin word for 'juice') contains fat, as well as protein, electrolytes, lymphocytes, and other substances. The incidence of

\footnotetext{
${ }^{1}$ Assistant Professor, ${ }^{2}$ Senior Resident

${ }^{3}$ Director and Professor, ${ }^{4}$ Professor

1,2,4 Department of Endocrine and Breast Surgery, Sanjay Gandhi Post Graduate Institute of Medical Sciences, Lucknow Uttar Pradesh, India

${ }^{3}$ Department of Urology and Renal Transplantation, Sanjay Gandhi Post Graduate Institute of Medical Sciences, Lucknow Uttar Pradesh, India

Corresponding Author: Amit Agarwal, Professor, Department of Endocrine and Breast Surgery, Sanjay Gandhi Post Graduate Institute of Medical Sciences, Lucknow, Uttar Pradesh, India Phones: 8004904645, e-mail: amit@sgpgi.ac.in
}

chyle leaks is low, however, when they do occur, they can be difficult to manage and treat. Chyle fistula is caused by inadvertent injury of the thoracic duct, which is a rare but important complication (1 to $2.5 \%$ ) during neck dissection and can produce severe nutritional, metabolic, and immune disorders in the patient. ${ }^{1}$

\section{ANATOMICAL CONSIDERATIONS}

There is considerable anatomical variation in the cervical portion of the thoracic duct, ${ }^{2}$ although it usually courses along the medial border of the scalenus medius muscle. The terminal arch of the duct can be between 0.5 and $4 \mathrm{~cm}$ above the clavicle before one or multiple thoracic lymphatic vessels join the venous system, most commonly the internal jugular vein. These ducts may be very thin-walled and delicate. In a study of 24 cadavers, Langford et $\mathrm{al}^{3}$ found that the thoracic duct approached the venous angle (the junction of the internal jugular and subclavian vein) posteriorly, terminating within $1 \mathrm{~cm}$ of this landmark. Studies show marked variability both in the anatomy and termination of the thoracic duct; therefore, it is not surprising that it is subject to damage during low-neck dissection surgery as well as in some selective neck dissections. ${ }^{4}$ The variation in the anatomy makes this structure more prone for damage (Figs 1 and 2).

\section{NUTRITIONAL MANAGEMENT}

The primary goals of nutritional management of chyle leaks are to decrease production of chyle fluid in order to avoid aggravating the excessive drainage, replace fluid and electrolytes; and maintain or replete nutritional status and prevent malnutrition.

Nutritional options for treatment include nutrition intervention, such as an essentially fat free diet; fat free diet supplemented with medium chain triglycerides (MCTs), specialized enteral feeding (fat free, MCT based or very low fat); or total parenteral nutrition (TPN). ${ }^{5}$

Some authors suggest introducing a high fat meal in patients in whom the chyle leak is believed to be closed, ensuring closure before removal of drainage tubes. ${ }^{6}$ It is virtually impossible to eliminate all fat from the diet and such a diet may be difficult to maintain, unless the patient is extremely motivated and compliant. Fat-soluble vitamin and essential fatty acid supplementation, or supplemental nutrition support need to be addressed. 


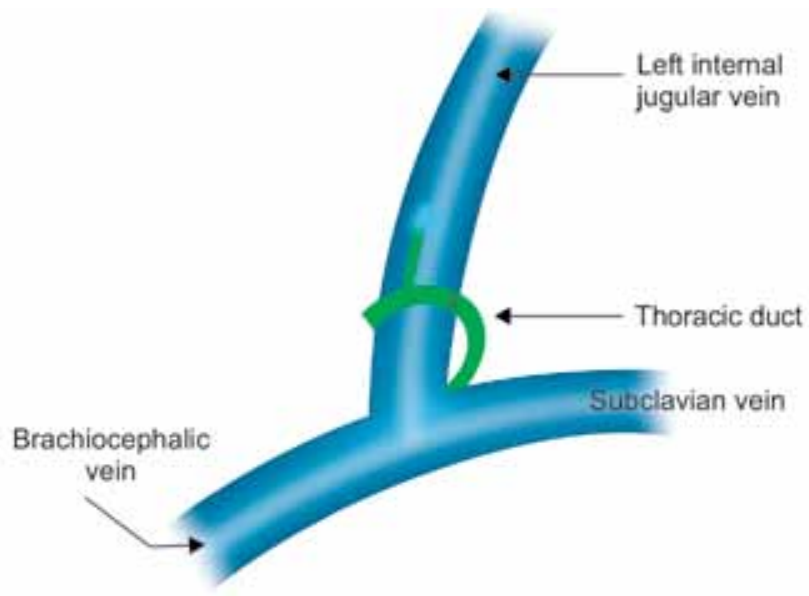

Fig. 1: Thoracic duct according to classical description

A therapeutic vitamin and mineral supplement may be necessary to ensure complete nutritional intake.

\section{MEDIUM CHAIN TRIGLYCERIDES (MCTS)}

Medium chain triglycerides are often recommended in the treatment of chyle leak and are thought to be absorbed directly across the mucosa into the portal circulation and does not require transport via the lymph system.

Medium chain triglycerides is available as MCT oil or in specialized oral/enteral supplements and contains 8.3 calories per gram $(1 \mathrm{~Tb}=15 \mathrm{ml}=115 \mathrm{kcal})$. Unfortunately, MCT oil is unpalatable and not generally well accepted by patients. Too much may cause diarrhea and gastrointestinal distress. Doses of 60 to 70 grams / day (4 to 5 tablespoons; 500 to 600 calories) spread out throughout the day are generally tolerated. Patients following a fat free or diet with MCT oil as the only fat source for any length of time may need to supplement essential fatty acids (EFA) and fat soluble vitamins. ${ }^{7}$

\section{NEGATIVE PRESSURE SUCTION DRESSING}

Negative pressure suction dressing involves covering and sealing the wound with a drape and applying subatmospheric pressure. This results in various effects, such as wound shrinkage, increase in blood flow, increase in granule formation, and removal of fluids, which lead to early wound closure. ${ }^{8,9}$ Negative pressure suction dressing is also reported to be effective for postoperative fistula-accompanied wounds, such as enterocutaneous fistula, ${ }^{10}$ bronchopleural fistula, ${ }^{11}$ and groin lymphatic fistula, ${ }^{12}$ although there is no report on negative pressure suction dressing for chylous fistula after neck dissection.

\section{Octreotide}

Somatostatin and its long-acting analog octreotide were used by Ulibarri et $\mathrm{al}^{13}$ as an effective and successful

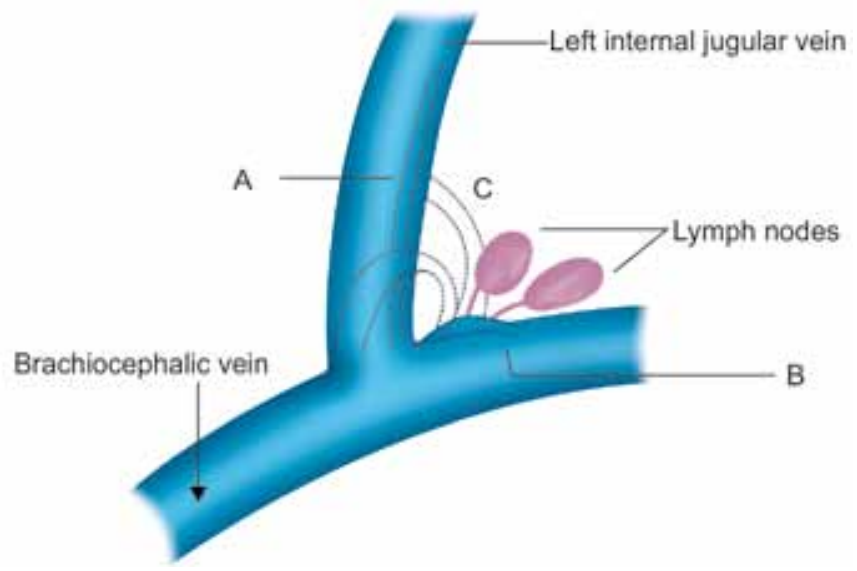

Fig. 2: Thoracic duct variations [A: Single duct dividing into two channels, B: Abnormal lymphatic channels from lymph nodes and C: High opening of thoracic duct into IJV]

adjunct to conservative therapy of iatrogenic thoracic duct injuries. Later in a prospective study on dogs it was demonstrated a three-fold decrease in thoracic duct fistula output after administration of octreotide. ${ }^{14}$ Somatostain is a peptide that acts both as a neurohormone and paracrine agent. Its biologic actions are extremely diverse and include the inhibition of thyroid-stimulating hormone, growth hormone, vasoactive intestinal peptide, gastrin, motilin, insulin, glucagon, intestinal secretions, and bile flow. Although the exact mechanism of action of octreotide in chyle leaks is not well-defined, it is attributed to its ability to reduce gastrointestinal and pancreatic secretions, decrease hepatic venous pressure, and reduce splanchnic blood flow. ${ }^{15}$ Doses begin at $50 \mathrm{mcg}$ subcutaneously three times daily and can be increased up to $200 \mathrm{mcg}$ three times daily. However, there is currently no consensus on when to start therapy, the most appropriate dose, increased incidence of gallstones secondary to decreased bile production and gallbladder contractility.

\section{Tranexamic Acid}

Tranexamic acid is a synthetic derivative of the amino acid lysine that exerts its antifibrinolytic effect through the reversible blockade of lysine binding sites on plasminogen molecules. Intravenously administered tranexamic acid caused reductions in postoperative blood losses in patients undergoing surgery. Tranexamic acid is well tolerated; nausea and diarrhea are the most common adverse events. Increased risk of thrombosis with the drug has not been demonstrated in clinical trials. ${ }^{16}$

A few authors have reported that primary intestinal lymphangiectasia (PIL) patients responded to tranexamic acid ( $1 \mathrm{gm}, 3$ times a day) but these responses were heterogeneous with only partial disease attenuation. It was hypothesized that increasing plasma fibrinolysis 


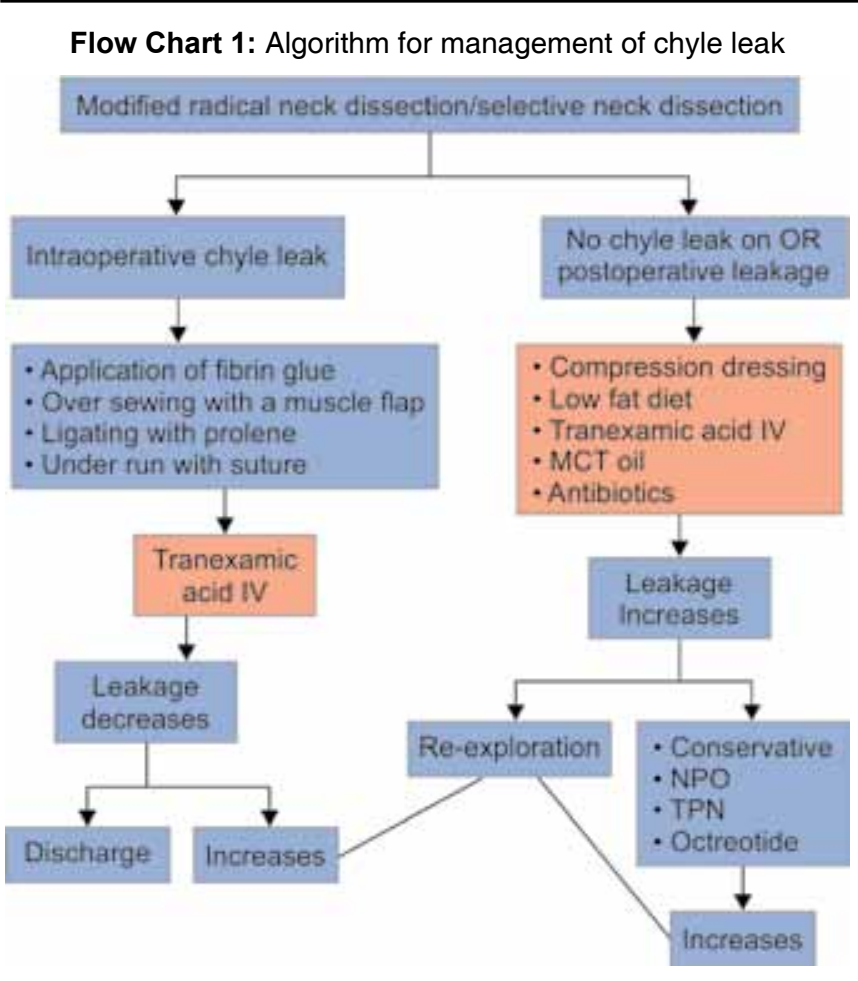

might enhance lymphatic permeability to plasma proteins. Tranexamic acid was found to reduce chylous ascites following retroperitoneal lymph node dissections. ${ }^{17}$ Tranexamic acid reduces the local fibrinolysis and also postulated to prevent the rupture of lymphatic-venous anastomosis which usually occurs when there is increased lymphatic pressure. ${ }^{18}$

\section{CONCLUSION}

Chyle leak is a form of morbidity which is difficult to manage. To prevent we recommend that the surgeon should have clear knowledge of anatomical variations of the thoracic duct and fine tissue handling in the region of the thoracic duct. Majority of the chyle leaks respond to the usual conservative management and by adding tranexamic acid to the armamentarium, now majority of chyle leaks close spontaneously, if other factors are taken care of. We provide our algorithm of conservative management of chyle leak (Flow Chart 1).

\section{REFERENCES}

1. Crumley RL, Smith JD. Postoperative chylous fistula prevention and management. Laryngoscope 1976 Jun;86(6): 804-813.
2. Kinnaert P. Anatomical variations of the cervical portion of the thoracic duct in man. J Anat 1973 May;115(Pt 1):45-52.

3. Langford RJ, Daudia AT, Malins TJ. A morphological study of the thoracic duct at the jugulo-subclavian junction. J Craniomaxillofac Surg 1999 Apr;27(2):100-104.

4. Roh JL, Yoon YH, Park CI. Chyle leakage in patients undergoing thyroidectomyplus central neck dissection for differentiated papillary thyroid carcinoma. Ann Surg Oncol 2008 Sep;15(9):2576-2580.

5. Myers EN, Dinerman WS. Management of chylous fistulas. Laryngoscope 1975 May;85(5):835-840.

6. Golden $\mathrm{P}$, et al. Chylothorax in blunt trauma: a case report. Am J Crit Care 1999;8(3):189-192.

7. Baumgartner T. Parenteral Macronutrition. In: Baumgartner T, editor. Clinical Guide to Parenteral Micronutrition. USA: Fujisawa; 1997:47.

8. Argenta LC, Morykwas MJ. Vacuum-assisted closure: a new method for wound control and treatment: clinical experience. Ann Plast Surg 1997 Jun;38(6):563-576; discussion 577.

9. Orgill DP, Bayer LR. Update on negative-pressure wound therapy. Plast Reconstr Surg 2011;127(suppl 1):105S-115S.

10. Goverman J, Yelon JA, Platz JJ, Singson RC, Turcinovic M. The 'Fistula VAC,' a technique for management of enterocutaneous fistulae arising within the open abdomen: report of 5 cases. J Trauma 2006 Feb;60(2):428-431; discussion 431.

11. Harada A, Nakamura Y, Fukumori K, Nagata T, Iguro Y. Negative pressure wound therapy was useful in treating empyema with bronchopleural fistula. Kyobu Geka. Japanese J Thor Sur 2010 Nov;63(12):1039-1043.

12. Lemaire V, Brilmaker J, Kerzmann A, Jacquemin D. Treatment of a groin lymphatic fistula with negative pressure wound therapy. Eur J Vasc Endovasc Surg 2008 Oct 31;36(4):449-451.

13. Ulibarri JI, Sanz Y, Fuentes C, Mancha A, Aramendia M, Sanchez S. Reduction of lymphorrhagia from ruptured thoracic duct by somatostatin. Lancet 1990 Jul 28;336(8709):258.

14. Markham KM, Glover JL, Welsh RJ, Lucas RJ, Bendick PJ. Octreotide in the treatment of thoracic duct injuries. Am Surg 2000 Dec 1;66(12):1165-1167.

15. Davis SN, Granner DK. Insulin, oral hypoglycemic agents, and the pharmacology of the endocrine pancreas. In: Gilman AG, Hardman JG, Limbird LE, et al, editors. Goodman and Gilman's the pharmacological basis of therapeutics, 9th ed. New York, NY: Pergamon Press; 1996. p. 1512-1513.

16. Dunn CJ, Goa KL. Tranexamic acid: a review of its use in surgery and other indications. Drugs. 1999 Jun;57(6):1005-1032. Review. PubMed PMID: 10400410.

17. Leibovitch I, Mor Y, Golomb J, Ramon J. The diagnosis and management of postoperative chylous ascites. J Urol $2002 \mathrm{Feb}$ 28;167(2):449-457.

18. Bliss CM, Schroy PC. Primary intestinal lymphangiectasia. Current treatment options in gastroenterology 2004 Feb 1; 7(1):3-6. 